\title{
DIFFUSIVE SHOCK ACCELERATION IN TEST-PARTICLE REGIME
}

\author{
Hyesung KANG ${ }^{1}$ and Dongsu RYu ${ }^{2}$ \\ ${ }^{1}$ Department of Earth Sciences, Pusan National University, Pusan 609-735, Republic of Korea; kang@uju.es.pusan.ac.kr \\ ${ }^{2}$ Department of Astronomy and Space Science, Chungnam National University, Daejeon 305-764, Republic of Korea; ryu @ canopus.cnu.ac.kr \\ Received 2010 July 2; accepted 2010 July 30; published 2010 September 1
}

\begin{abstract}
We examine the test-particle solution for diffusive shock acceleration, based on simple models for thermal leakage injection and Alfvénic drift. The critical injection rate, $\xi_{c}$, above which the cosmic-ray (CR) pressure becomes dynamically significant depends mainly on the sonic shock Mach number, $M$, and preshock gas temperature, $T_{1}$. In the hot-phase interstellar medium (ISM) and intracluster medium, $\xi_{c} \lesssim 10^{-3}$ for shocks with $M \lesssim 5$, while $\xi_{c} \approx 10^{-4}\left(T_{1} / 10^{6} \mathrm{~K}\right)^{1 / 2}$ for shocks with $M \gtrsim 10$. For $T_{1}=10^{6} \mathrm{~K}$, for example, the test-particle solution would be valid if the injection momentum $p_{\text {inj }}>3.8 p_{\text {th }}$ (where $p_{\text {th }}$ is thermal momentum). This leads to a postshock CR pressure less than $10 \%$ of the shock ram pressure. If the Alfvén speed is comparable to the sound speed in the preshock flow, as in the hot-phase ISM, the power-law slope of CR spectrum can be significantly softer than the canonical test-particle slope. Then, the CR spectrum at the shock can be approximated by the revised test-particle power law with an exponential cutoff at the highest accelerated momentum, $p_{\max }(t)$. An analytic form of the exponential cutoff is also suggested.
\end{abstract}

Key words: acceleration of particles - cosmic rays - shock waves

Online-only material: color figures

\section{INTRODUCTION}

Suprathermal particles are produced as an inevitable consequence of the formation of collisionless shocks in tenuous astrophysical plasmas and they can be further accelerated to very high energies through interactions with resonantly scattering Alfvén waves in the converging flow across a shock (Bell 1978; Drury 1983; Blandford \& Eichler 1987; Malkov \& Drury 2001). The most attractive feature of the diffusive shock acceleration (DSA) theory is the simple prediction of the power-law momentum distribution of cosmic rays (CRs), $f(p) \propto p^{-3 \sigma /(\sigma-1)}$ (where $\sigma$ is the shock compression ratio), in the test-particle regime. For strong, adiabatic gas shocks, this gives a power-law index of 4 , which is reasonably close to the observed, "universal" index of the CR spectra in many environments.

The nonthermal particle injection and ensuing acceleration at shocks depend mainly upon the shock Mach number, field obliquity angle, and the strength of the Alfvén turbulence responsible for scattering. At quasi-parallel shocks, the shock Mach number is the primary parameter that determines the CR acceleration efficiency, while the injection fraction, $\xi$ (the ratio of CR particles to the total particles passed through the shock), is the secondary parameter. Detailed nonlinear treatments of DSA predict that at strong shocks, with a small fraction of $\xi>10^{-4}$, a significant fraction of the shock kinetic energy is transferred to CRs and there are highly nonlinear back-reactions from CRs to the underlying flow (Berezhko \& Völk 1997; Kang \& Jones 2007). Indeed, multi-band observations of nonthermal radio to $\gamma$-ray emissions from several supernova remnants (SNRs) have been successfully explained by efficient DSA features such as high degree of shock compression and amplification of magnetic fields in the precursor (e.g., Reynods 2008; Berezhko et al. 2009; Morlino et al. 2009).

It has been recognized, however, that the $\mathrm{CR}$ spectrum at sources, $N(E)$, predicted for shocks strongly modified by CR feedback may be too flat to be consistent with the observed flux of CR nuclei at Earth, $J(E)$. Recently, Ave et al. (2009) analyzed the spectrum of CR nuclei up to $\sim 10^{14} \mathrm{eV}$ measured by TRACER instruments and found that the CR spectra at Earth can be fitted by a single power law of $J(E) \propto E^{-2.67}$. Assuming an energy-dependent propagation path length $\left(\Lambda \propto E^{-0.6}\right)$, they suggested that a soft source spectrum, $N(E) \propto E^{-s}$ with $s \sim 2.3-2.4$, is preferred by the observed data. This is much softer than the CR spectrum that the nonlinear DSA predicts for strong SNRs, which are believed to be the main accelerators for Galactic CRs up to the knee energy around $10^{15.5} \mathrm{eV}$. Thus, in order to reconcile the DSA prediction with the observed $J(E)$, the bulk of Galactic CRs should originate from SNRs in which the CR acceleration efficiency is $10 \%$ or so (i.e., roughly in the test-particle regime). Such inefficient acceleration could be possible for SNRs in the hot phase of the interstellar medium (ISM; i.e., low shock Mach number shocks) and for the inject fraction smaller than $10^{-4}$ (Kang 2010).

The scattering by Alfvén waves tends to isotropize the CR distribution in the wave frame, which may drift upstream at Alfvén speed with respect to the bulk plasma (Skilling 1975). This Alfvénic drift in the upstream region reduces the velocity jump that the particles experience across the shock, which in turn softens the CR spectrum beyond the canonical testparticle slope ( $s=2$ for strong shocks; Kang 2010; Caprioli et al. 2010). Moreover, the Alfvénic drift in amplified magnetic fields both upstream and downstream can drastically soften the accelerated particle spectrum even in nonlinear modified shocks (Zirakashvili \& Ptuskin 2008; Ptuskin et al. 2010).

At collisionless shocks, suprathermal particles moving faster than the postshock thermal distribution may swim through the MHD waves and leak upstream across the shocks and get injected into the CR population (Malkov 1998; Gieseler et al. 2000; Kang et al. 2002). But it is not yet possible to make precise quantitative predictions for the injection process from first principles, because complex plasma interactions among CRs, waves, and the underlying gas flow are not fully understood yet (e.g., Malkov \& Drury 2001). Until plasma simulations such as hybrid or particle-in-cell simulations reach 

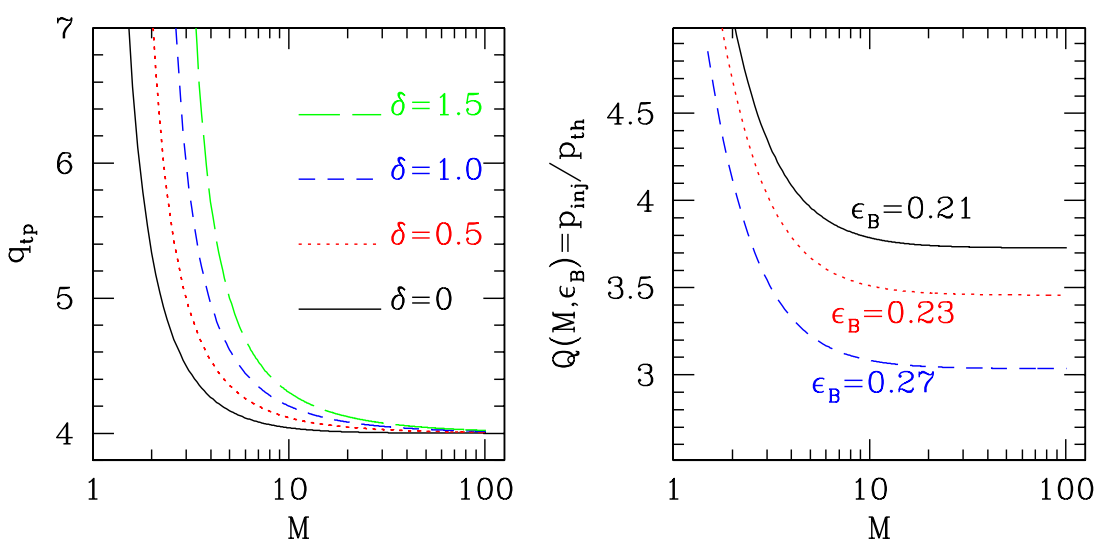

Figure 1. Left: test-particle power-law slope, $q_{\mathrm{tp}}$, revised by including the Alfvénic drift (Equation (2)), is shown as a function of the sonic Mach number for four different values of $\delta=v_{A} / c_{s}$. Right: ratio $Q_{\text {inj }}=p_{\text {inj }} / p_{\text {th }}$ is shown for three values of $\epsilon_{B}=B_{0} / B_{\perp}=0.21,0.23$, and 0.27.

(A color version of this figure is available in the online journal.)

the stage where the full problem can be treated with practical computational resources, in the studies of DSA we have to adopt a phenomenological injection scheme that can emulate the injection process.

In this paper, we will examine the relation between the thermal leakage injection model described in Kang et al. (2002) and the time-dependent test-particle solutions for DSA. The basic models are described in Section 2, while the analytic expression for the CR spectrum in the test-particle limit is suggested in Section 3. Finally, a brief summary will be given in Section 4.

\section{BASIC MODELS}

In the kinetic DSA approach, the following diffusionconvection equation for the pitch-angle-averaged distribution function, $f(x, p, t)$, is solved along with suitably modified gasdynamic equations:

$$
\frac{\partial f}{\partial t}+\left(u+u_{w}\right) \frac{\partial f}{\partial x}=\frac{p}{3} \frac{\partial\left(u+u_{w}\right)}{\partial x} \frac{\partial f}{\partial p}+\frac{\partial}{\partial x}\left[\kappa(x, p) \frac{\partial f}{\partial x}\right],
$$

where $\kappa(x, p)$ is the spatial diffusion coefficient and $u_{w}$ is the drift speed of the local Alfvénic wave turbulence with respect to the plasma (Skilling 1975). We consider only the proton CR component.

\subsection{Alfvénic Drift Effect}

Since the Alfvén waves upstream of the subshock are expected to be established by the streaming instability, the wave speed is set there to be $u_{w}=-v_{A}$. Downstream, it is likely that the Alfvénic turbulence is nearly isotropic, hence $u_{w}=0$ there. As a result, the velocity jump across the shock is reduced, and the slope of the test-particle power-law spectrum should be revised as

$$
q_{\mathrm{tp}}=\frac{3\left(u_{1}-v_{A}\right)}{u_{1}-v_{A}-u_{2}}=\frac{3 \sigma\left(1-M_{A}^{-1}\right)}{\left(\sigma-1-\sigma M_{A}^{-1}\right)},
$$

where $u_{1}$ and $u_{2}$ are the upstream and downstream speeds, respectively, in the shock rest frame, $\sigma=u_{1} / u_{2}=\rho_{2} / \rho_{1}$ is the shock compression ratio, and $v_{A}$ and $M_{A}=u_{1} / v_{A}$ are the Alfvén speed upstream and the Alfvén Mach number, respectively. Hereafter, we use the subscripts " 1 ," and " 2 " to denote conditions upstream and downstream of the shock, respectively. Thus, the $\mathrm{CR}$ spectrum would be softer than the canonical power-law spectrum with the slope, $3 \sigma /(\sigma-1)$, unless $M_{A} \gg 1$.

The left panel of Figure 1 shows the revised test-particle slope $q_{\text {tp }}$ as a function of the sonic Mach number, $M$, for different Alfvén speeds, $v_{A}=\delta \cdot c_{s}$ (where $c_{s}$ is the upstream sound speed). In the hot-phase ISM of $T \approx 10^{6} \mathrm{~K}$ with hydrogen number density $n_{H} \approx 0.003 \mathrm{~cm}^{-3}$ and magnetic field strength $B \approx 5 \mu \mathrm{G}$, the sound speed is $c_{s} \approx 150 \mathrm{~km} \mathrm{~s}^{-1}$ and the Alfvén speed is $v_{A} \approx 170 \mathrm{~km} \mathrm{~s}^{-1}$. So, $\delta \approx 1$ is a representative value. If $\delta \approx\left(P_{B} / P_{g}\right)^{1 / 2} \approx 1$, the Alfvén drift effect is significant for the Alfvén Mach number, $M_{A} \approx M \lesssim 30$. Consequently, this effect reduces the $\mathrm{CR}$ acceleration efficiency. Of course, it is not important for strong shocks with $u_{s} \gg c_{s} \sim v_{A}$ (i.e., $M_{A} \gtrsim 30$ ).

\subsection{Thermal Leakage Injection Model}

Since the velocity distribution of suprathermal particles is not isotropic in the shock frame, the diffusion-convection equation cannot directly follow the injection from the non-diffusive thermal pool into the diffusive CR population. Here, we adopt the thermal leakage injection model that was originally formulated by Gieseler et al. (2000) based on the calculations of Malkov (1998). In this model, particles above a certain injection momentum $p_{\text {inj }}$ cross the shock and get injected to the CR population. We adopt a smooth "transparency function," $\tau_{\mathrm{esc}}\left(\epsilon_{B}, v\right)$, that expresses the probability of suprathermal particles at a given velocity, $v$, leaking upstream through the postshock MHD waves. One free parameter controls this function: $\epsilon_{B}=B_{0} / B_{\perp}$, the ratio of the general magnetic field along the shock normal, $B_{0}$, to the amplitude of the postshock MHD wave turbulence, $B_{\perp}$. Although plasma hybrid simulations and theories both suggested that $0.25 \lesssim \epsilon_{B} \lesssim 0.35$ (Malkov \& Völk 1998), the physical range of this parameter remains rather uncertain due to lack of full understanding of relevant plasma interactions. Since $\tau_{\text {esc }}$ increases gradually from zero to one in the thermal tail distribution, the "effective" injection momentum can be approximated by

$$
p_{\text {inj }} \approx 1.17 m_{p} u_{2}\left(1+\frac{1.07}{\epsilon_{B}}\right) \equiv Q_{\text {inj }}\left(M, \epsilon_{B}\right) p_{\text {th }},
$$

where $p_{\text {th }}=\sqrt{2 m_{p} k_{B} T_{2}}$ is the thermal peak momentum of the immediate postshock gas with temperature $T_{2}$ and $k_{B}$ is the Boltzmann constant (Kang et al. 2002). 
The right panel of Figure 1 shows the value of $Q_{\text {inj }}$ as a function of $M$ for three values of $\epsilon_{B}=0.21,0.23$, and 0.27 , which represent "inefficient," "moderately efficient," and "efficient" injection cases, respectively (see Figure 4 below). At weaker shocks, the compression is smaller and so the ratio $u_{2} / u_{1}$ is larger. For stronger turbulence (larger $B_{\perp}$, smaller $\epsilon_{B}$ ), it is harder for particles to swim across the shock. So for both of these cases, $p_{\text {inj }}$ has to be larger. Hence, the value of $Q_{\text {inj }}\left(M, \epsilon_{B}\right)$ is larger for weaker shocks and for smaller $\epsilon_{B}$, which leads to a lower injection fraction.

In our thermal leakage injection model, the CR distribution function at $p_{\text {inj }}$ is then anchored to the postshock Maxwellian distribution as

$$
f_{\text {inj }}=f\left(p_{\text {inj }}\right)=\frac{n_{2}}{\pi^{1.5}} p_{\text {th }}^{-3} \exp \left(-Q_{\text {inj }}^{2}\right),
$$

where $n_{2}$ is the postshock proton number density and the distribution function is defined in general as $\int 4 \pi p^{2} f(p) d p=n$. For the test-particle power-law spectrum, the value of $Q_{\text {inj }}$ determines the amplitude of the subsequent suprathermal power-law distribution as $f(p)=f_{\text {inj }} \cdot\left(p / p_{\text {inj }}\right)^{-q_{\mathrm{tp}}}$. Then, the CR injection fraction can be defined as

$$
\xi \equiv \frac{n_{\mathrm{CR}}}{n_{2}}=\frac{4}{\sqrt{\pi}} Q_{\mathrm{inj}}^{3} \exp \left(-Q_{\mathrm{inj}}^{2}\right) \frac{1}{q_{\mathrm{tp}}(M)-3},
$$

which depends only on the ratio $Q_{\text {inj }}$ and the slope $q_{\mathrm{tp}}$, but not on the postshock temperature $T_{2}$. For $Q_{\text {inj }}=3.8$, for example, $\xi=6.6 \times 10^{-5} /\left(q_{\text {tp }}-3\right)$, which becomes $\xi=6.6 \times 10^{-5}$ for strong shocks with $q_{\text {tp }}=4.0$.

\subsection{Bohm-type Diffusion Model}

In modeling DSA, it is commonly assumed that the particles are resonantly scattered by self-generated waves, so the Bohm diffusion model can represent a saturated wave spectrum (i.e., the mean scattering length, $\lambda=r_{g}$, where $r_{g}$ is the gyroradius). Here, we adopt a Bohm-type diffusion coefficient that includes a weaker non-relativistic momentum dependence,

$$
\kappa(x, p)=\kappa^{*} \cdot\left(\frac{p}{m_{p} c}\right)^{\alpha}\left[\frac{\rho(x)}{\rho_{1}}\right]^{-m},
$$

where the coefficient $\kappa^{*}=m_{p} c^{3} /\left(3 e B_{0}\right)$ depends on the upstream mean field strength. The case with $m=1$ approximately accounts for the compressive amplification of Alfvén waves.

The mean acceleration time for a particle to reach $p_{\max }$ from $p_{\text {inj }}$ in the test-particle limit of DSA theory can be approximated by

$$
t_{\mathrm{acc}}=\frac{3}{u_{1}-v_{A}-u_{2}} \int_{p_{\mathrm{inj}}}^{p_{\max }}\left(\frac{\kappa_{1}}{u_{1}-v_{A}}+\frac{\kappa_{2}}{u_{2}}\right) \frac{d p}{p},
$$

if we assume the bulk drift of waves with $v_{A}$ in the upstream region (e.g., Drury 1983). Then, the maximum momentum can be estimated by setting $t=t_{\text {acc }}$ as

$$
p_{\max }(t)^{\alpha} \approx \frac{\alpha\left(1-M_{A}^{-1}\right)\left(\sigma-1-\sigma M_{A}^{-1}\right)}{3 \sigma\left[1+\left(1-M_{A}^{-1}\right) \sigma^{1-m}\right]} \frac{u_{s}^{2}}{\kappa^{*}} t=f_{c} \frac{u_{s}^{2}}{\kappa^{*}} t,
$$

where $u_{s}=u_{1}$ is the shock speed (Kang et al. 2009). For the case of $m=1$, the typical value of the parameter, $f_{c}=\alpha\left(1-M_{A}^{-1}\right)\left(\sigma-1-\sigma M_{A}^{-1}\right) /\left\{3 \sigma\left[1+\left(1-M_{A}^{-1}\right) \sigma^{1-m}\right]\right\}$, is $\sim 1 / 8$ in the limit of $M_{A} \gg 1$ and $M \gg 1$.

\section{TEST-PARTICLE SPECTRUM}

If the injection is inefficient, especially at weak shocks, the CR pressure remains dynamically insignificant and the test-particle solution is valid. Caprioli et al. (2009, hereafter CBA09) derived the analytic solution for a steady-state, test-particle shock with a free-escape boundary (FEB) at a distance $x_{\mathrm{FEB}}$ upstream of the shock (i.e., $\left.f\left(x>x_{\mathrm{FEB}}\right)=0\right)$. For a diffusion coefficient that depends on the momentum as $\kappa(p)=\kappa^{*}\left(p / m_{p} c\right)^{\alpha}$, the CR distribution at the shock location, $x_{s}$, is given by

$$
f_{\mathrm{tp}}\left(x_{s}, p\right)=f_{0} \cdot \exp \left[-q_{\mathrm{tp}} \int_{z_{\mathrm{inj}}}^{z} \frac{d z^{\prime}}{z^{\prime}} \frac{1}{1-\exp \left(-1 / z^{\prime \alpha}\right)}\right],
$$

where $z=p / p^{*}, z_{\text {inj }}=p_{\text {inj }} / p^{*}, f_{0}=f_{\text {inj }}$, and $p^{*} / m_{p} c=$ $\left(x_{\mathrm{FEB}} u_{s} / \kappa^{*}\right)^{1 / \alpha}$ is the cutoff momentum set by the FEB. This expression can be rewritten as

$$
f_{\mathrm{tp}}\left(x_{s}, p\right)=f_{\mathrm{inj}} \cdot\left(\frac{p}{p_{\text {inj }}}\right)^{-q_{\mathrm{tp}}} \cdot \exp \left[-q_{\mathrm{tp}} C(z)\right],
$$

where the function $C(z)$ is given by

$$
C(z)=\int_{z_{\mathrm{inj}}}^{z} \frac{d z^{\prime}}{z^{\prime}} \frac{1}{\exp \left(1 / z^{\prime \alpha}\right)-1} .
$$

We show the function $C(z)$ for $\alpha=0.5$ and 1 in the left panel of Figure 2. For $z \ll 1, C(z)$ is small and so $\exp \left[-q_{\mathrm{tp}} C(z)\right]=1$, as expected. For $z \gg 1, C(z) \approx z^{\alpha}=\left(p / p^{*}\right)^{\alpha}$. But this regime $\left(p \gg p^{*}\right)$ is not really relevant, because the resulting $f_{\mathrm{tp}}\left(x_{s}, p\right)$ is extremely small. We are more interested in the exponential cutoff where $p \sim p^{*}$. Figure 2 shows that $C(z)$ increases much faster than $z^{\alpha}$ near $z \sim 1$. In fact, at $z \sim 1$, approximately $C(z) \approx 0.29 z^{2}$ for $\alpha=1$ and $C(z) \approx 0.58 z$ for $\alpha=1 / 2$. Thus, Equation (10) can be approximated by

$$
f_{\mathrm{tp}}\left(x_{s}, p\right) \approx f_{\mathrm{inj}} \cdot\left(\frac{p}{p_{\mathrm{inj}}}\right)^{-q_{\mathrm{tp}}} \cdot \exp \left[-\frac{0.29 q_{\mathrm{tp}}}{\alpha}\left(\frac{p}{p^{*}}\right)^{2 \alpha}\right] .
$$

Kang et al. (2009) showed that the shock structure and the CR spectrum of time-dependent, CR-modified shocks with ever increasing $p_{\max }(t)$ are similar to those of steady-state shocks with particles escaping through the upper momentum boundary, i.e., $f\left(p>p_{\mathrm{ub}}\right)=0$, if compared when $p_{\mathrm{max}}(t)=p_{\mathrm{ub}}$ (see their Figures 10 and 11). They also showed that the exponential cutoff in the form of $\exp \left[-k\left(p / p_{\max }\right)^{2 \alpha}\right]$ matches well the DSA simulation results for CR-modified shocks. In the same spirit, we suggest that Equation (10) could represent the CR spectrum at the shock location for time-dependent, testparticle shocks without particle escape, in which the cutoff momentum is determined by the shock age as in Equation (8), i.e., $p^{*} \sim p_{\max }(t)$.

The distribution function $f\left(x, p_{\max }\right)$ in the upstream region decreases roughly as $\exp \left[-x / l_{d}\left(p_{\max }\right)\right]$, where the diffusion length for $p_{\max }$ is

$$
l_{d}\left(p_{\max }\right)=\frac{\kappa\left(p_{\max }\right)}{u_{s}}=f_{c} u_{s} t
$$

CBA09 spectrum in Equation (10) was derived from the FEB condition of $f\left(x>x_{\mathrm{FEB}}, p\right)=0$ for steady-state shocks, while $f(x, p) \rightarrow 0$ only at $x \rightarrow \infty$ (upstream infinity) for timeevolving shocks without particle escape. So, we presume that 

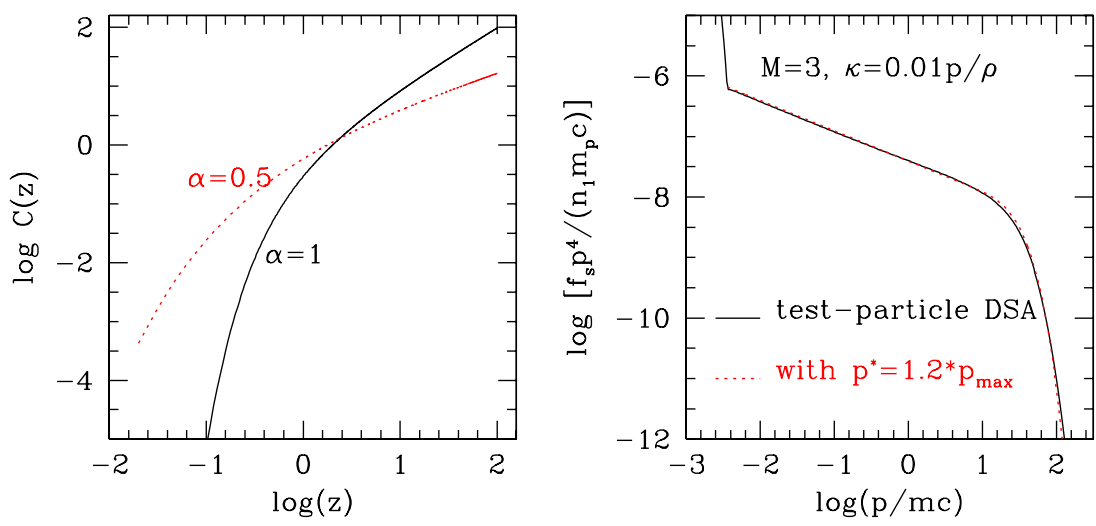

Figure 2. Left: function $C(z)$ defined in Equation (11) is shown as a function of $z \equiv p / p^{*}$ for $\kappa(p)=\kappa^{*}\left(p / m_{p} c\right)^{\alpha}$. Right: CR distribution at the shock position, $f_{s} \cdot p^{4}$ (in units of $n_{1} m_{p} c$ ), is shown for a Mach 3 shock. The solid line shows the results of a time-dependent DSA simulation without particle escape in the test-particle regime (i.e., no CR feedback to the flow). The dotted line represents the test-particle spectrum given in Equation (10) with $p^{*}=1.2 p_{\max }$.

(A color version of this figure is available in the online journal.)
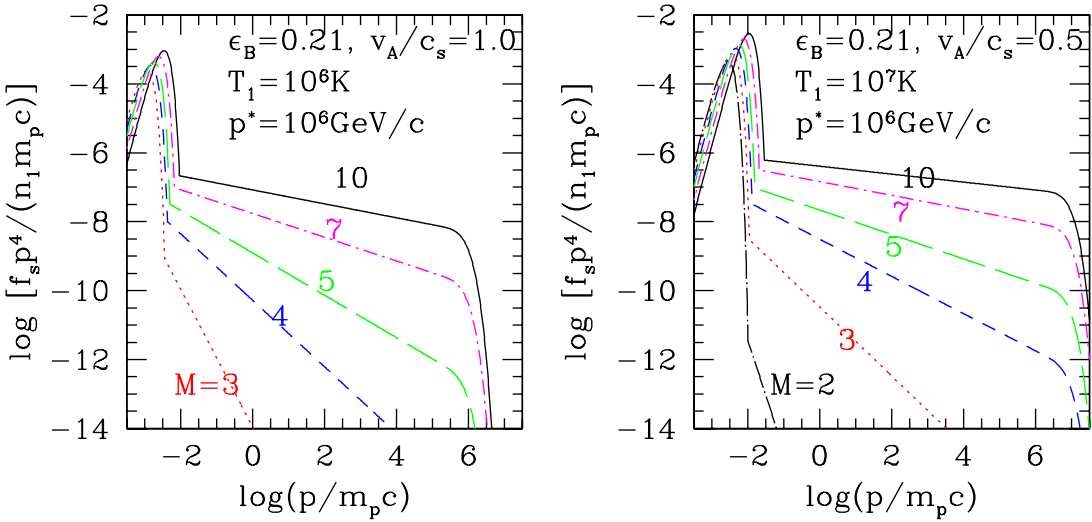

Figure 3. Left: test-particle spectra given in Equation (10) with $\epsilon_{B}=0.21, p^{*}=10^{6} \mathrm{GeV} / c, T_{1}=10^{6} \mathrm{~K}$, and $\delta=v_{A} / c_{s}=1.0$. Right: same as the left panel except $T_{1}=10^{7} \mathrm{~K}$ and $v_{A} / c_{s}=0.5$. Each curve is labeled with the shock Mach number, $M$, and the shock speed is $u_{s}=M \cdot 150 \mathrm{~km} \mathrm{~s}^{-1}\left(T_{1} / 10^{6} \mathrm{~K}\right)^{1 / 2}$.

(A color version of this figure is available in the online journal.)

the cutoff momentum can be found by setting the location of FEB at $x_{\mathrm{FEB}}=\zeta \cdot l_{d}\left(p_{\max }\right)$, where $\zeta \sim 1$. From the condition that $p^{*} / m_{p} c=\left(\zeta l_{d}\left(p_{\max }\right) u_{s} / \kappa^{*}\right)^{1 / \alpha}$, we find $p^{*}=\zeta \cdot p_{\max }$.

The right panel of Figure 2 shows the test-particle solution from a time-dependent DSA simulation in which the dynamical feedback of the CR pressure was turned off. Contrary to the CBA09 case, no FEB is enforced in this simulation, so the shock does not approach a steady state, but instead evolves in time. As the CRs are accelerated to ever higher energies $\left(p_{\max } \propto t\right)$, the scale length of the CR pressure increases linearly with time, $l_{d}\left(p_{\max }\right) \propto u_{s} t$. So, the shock structure evolves in a self-similar fashion, depending only on the similarity variable, $x /\left(u_{s} t\right)$ (see Kang \& Jones 2007). By setting $p^{*}=1.2 p_{\max }(t)$ (i.e., $\zeta=1.2$ ) and also by adopting the value of $f_{\text {inj }}$ from the DSA simulation result, we calculated $f_{\mathrm{tp}}\left(x_{s}, p\right)$ according to Equation (10). As can be seen in the figure, the agreement between the numerical DSA results and the analytic approximation is excellent. Thus, we take Equation (10) as the test-particle spectrum from DSA, where $q_{\mathrm{tp}}, p_{\mathrm{inj}}, f_{\mathrm{inj}}$, and $p^{*} \approx 1.2 p_{\max }(t)$ are given by Equations (2), (3), (4), and (8), respectively.

Figure 3 shows some examples of the test-particle spectrum given in Equation (10). We consider the shocks propagating into the hot phase of the ISM of $T_{1}=10^{6} \mathrm{~K}$ or a typical intracluster medium (ICM) of $T_{1}=10^{7} \mathrm{~K}$. The shock speed is given by $u_{s}=$ $M \cdot c_{s}$, where the sound speed is $c_{s}=150 \mathrm{~km} \mathrm{~s}^{-1}\left(T_{1} / 10^{6} \mathrm{~K}\right)^{1 / 2}$. For all the cases, we assume a constant cutoff momentum, $p^{*}=10^{6} \mathrm{GeV} / c$, which is close to the knee energy in the Galactic CR spectrum. For typical hot-phase ISM, $\delta=v_{A} / c_{s} \approx 1$ as mentioned before. For typical ICM, $n_{H} \approx 10^{-3} \mathrm{~cm}^{-3}$ and $B \approx 1-5 \mu \mathrm{G}$, so $\delta \approx 0.5$ is taken here. For typical test-particle limit solutions, we adopt $\epsilon_{B}=0.21$ to specify $p_{\text {inj }}$ given in Equation (3), which determines the anchoring point where the test-particle power law begins. This choice of $\epsilon_{B}$ results in the injection rate $\xi \lesssim 10^{-4}$ and the postshock CR pressure $P_{c, 2} /\left(\rho_{1} u_{s}^{2}\right) \lesssim 0.1$. As can be seen in Figure 3, for stronger (faster) shocks, the postshock gas is hotter, the amplitude $f_{\text {inj }}$ is higher and the power-law spectrum is harder.

Then, the CR pressure at the shock position can be calculated by

$$
P_{c}\left(x_{s}\right)=\frac{4 \pi}{3} c \int_{p_{\text {inj }}}^{\infty} f_{\mathrm{tp}}\left(x_{s}, p\right) \frac{p^{4} d p}{\sqrt{p^{2}+\left(m_{p} c\right)^{2}}} .
$$

For strong shocks with $q_{\mathrm{tp}}=4$, with the test-particle spectrum in Equation (10), $P_{c} \propto f_{\text {inj }} p_{\text {inj }}^{4} \ln \left(p^{*} / m_{p} c\right)$. Then, with a constant cutoff $p^{*}, P_{c} \propto \exp \left(-Q_{\mathrm{inj}}^{2}\right) Q_{\mathrm{inj}}^{4} p_{\mathrm{th}}$. So, for a fixed value of $Q_{\text {inj }}$ (or fixed injection fraction $\xi$ ), $P_{c} \propto p_{\text {th }} \propto u_{s}$. Figure 4 shows the fraction of injected particles and the postshock CR pressure calculated by adopting the test-particle spectrum given in Equation (10). The same $p^{*}=10^{6} \mathrm{GeV} / c$ is chosen as in Figure 3 . The quantities, $n_{\mathrm{cr}, 2}$ and $P_{c, 2}$ do not depend sensitively on the assumed value of $p^{*}$ for weak shocks, since the power 

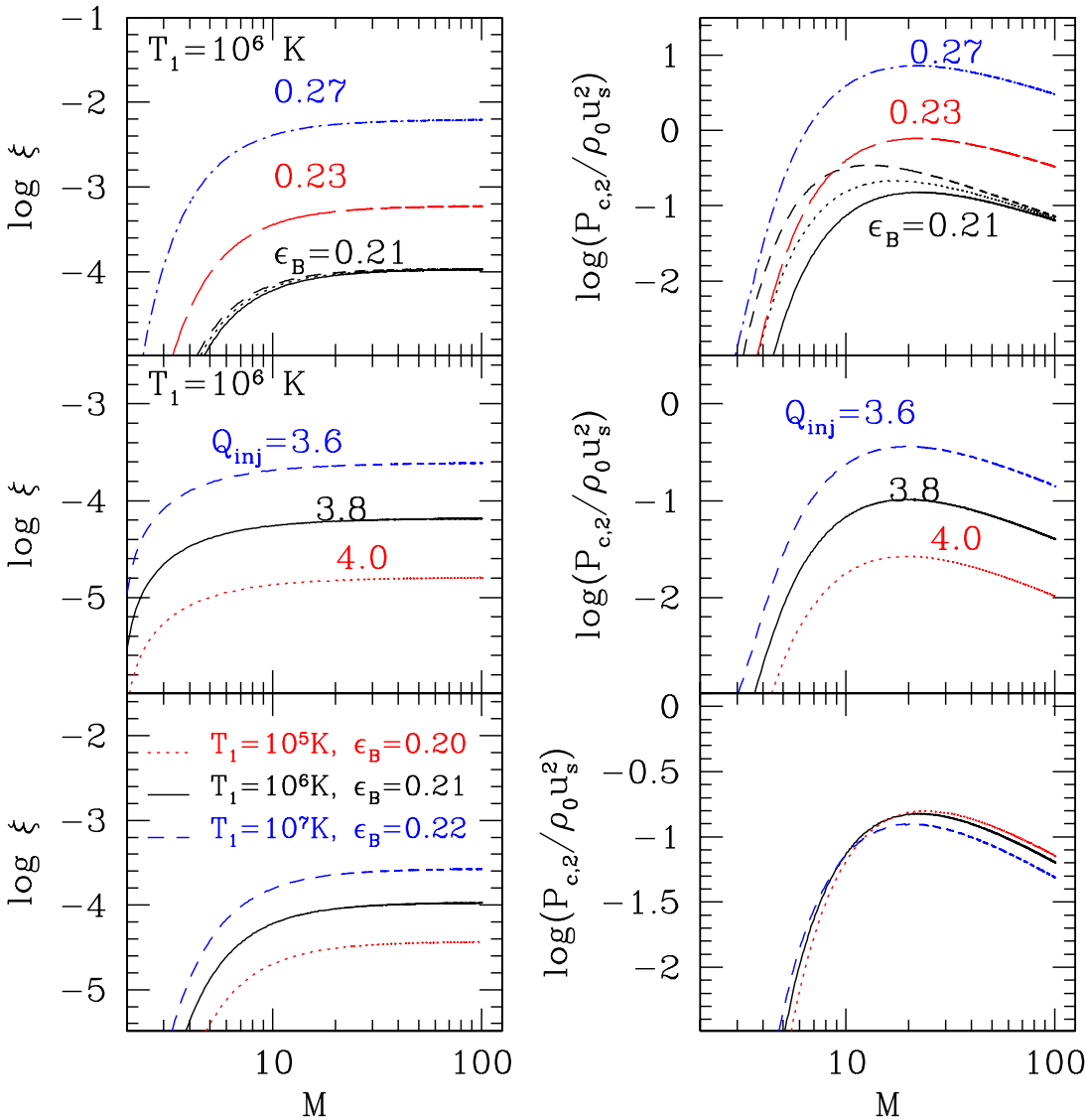

Figure 4. Fraction of CR particles, $\xi=n_{\mathrm{cr}, 2} / n_{2}$ (left panels), and the downstream CR pressure in units of the shock ram pressure (right panels) are shown for the test-particle spectrum given in Equation (10) with a fixed $p^{*}=10^{6} \mathrm{GeV} / c$. The shock speed is specified by $u_{s}=M \cdot 150 \mathrm{~km} \mathrm{~s}{ }^{-1}\left(T_{1} / 10^{6} \mathrm{~K}\right)^{1 / 2}$. Upper panels: shocks with the preshock temperature, $T_{1}=10^{6} \mathrm{~K}$. Three values of $\epsilon_{B}=0.21,0.23$ (long dashed lines), and 0.27 (dot dashed) are considered with $\delta=v_{A} / c_{s}=1.0$. For the case with $\epsilon_{B}=0.21$, three cases with $\delta=0$ (dashed lines), 0.5 (dotted), and 1.0 (solid) are shown. Middle panels: the same model shocks as the upper panels except a constant ratio $Q_{\text {inj }}=p_{\text {inj }} / p_{\text {th }}=3.6$ (dashed lines), 3.8 (solid), 4.0 (dotted) are shown. The Alfvén speed is $v_{A} / c_{s}=1.0$. Lower panels: shocks propagating into different temperature media, $T_{1}=10^{5} \mathrm{~K}$ (with $\epsilon_{B}=0.20$, dotted lines), $10^{6} \mathrm{~K}$ (with $\epsilon_{B}=0.21$, solid lines), and $10^{7} \mathrm{~K}$ (with $\epsilon_{B}=0.22$, dashed lines) are shown The Alfvén speed is $v_{A} / c_{S}=1.0$ for all three cases.

(A color version of this figure is available in the online journal.)

slope $q_{\mathrm{tp}}$ is greater than 4 . But for strong shocks $(M \gtrsim 30)$ where $q_{\mathrm{tp}} \approx 4$ (see Figure 1 ), the CR pressure increases logarithmically as $P_{c} \propto \ln \left(p^{*} / m_{p} c\right)$. Several values of $T_{1}, \epsilon_{B}$ (or $\left.Q_{\mathrm{inj}}\right)$, and $\delta=v_{A} / c_{s}$ are considered. In general, for fixed values of $\epsilon_{B}$ (or $Q_{\text {inj }}$ ) and $\delta$, the ratio $P_{c, 2} /\left(\rho_{1} u_{s}^{2}\right)$ increases strongly with the shock Mach number for shocks with $M \lesssim 10$ because of the strong dependence of $\xi$ (or $Q_{\mathrm{inj}}$ ) on $M$ for weaker shocks. But for shocks with $M>10, \xi$ becomes independent of $M$ and so $P_{c} \propto u_{s}$, as discussed above. So, the CR pressure relative to the shock ram pressure $P_{c, 2} /\left(\rho_{1} u_{s}^{2}\right) \propto u_{s}^{-1}$, that is, it becomes smaller at faster shocks. Of course, in the nonlinear DSA regime, the ratio $P_{c, 2} /\left(\rho_{1} u_{s}^{2}\right)$ increases with the shock Mach number and saturates at about $1 / 2$ (Kang et al. 2009).

The top panels of Figure 4 show how the CR pressure depends on $\epsilon_{B}$ and $\delta$. For a given Mach number, the CR pressure increases strongly with $\epsilon_{B}$, because of the $\exp \left(-Q_{\mathrm{inj}}^{2}\right)$ factor. Obviously, the CR pressure becomes smaller for larger $\delta$ because of softer power-law spectra at weaker shocks with $M \lesssim 30$. For $\epsilon_{B}=0.21$ and $\delta=1, \xi \lesssim 10^{-4}$ and $P_{c, 2} /\left(\rho_{1} u_{s}^{2}\right) \lesssim 0.1$, so the test-particle solution would provide a good approximation. For $\epsilon_{B}=0.23$, on the other hand, the injection fraction becomes $\xi \approx 10^{-4}$ to $10^{-3}$, and the test-particle solution is no longer valid for $M \gtrsim 5$. For weak cosmological shocks with $M \lesssim 3$, typically found in the hot ICM (e.g., Ryu et al. 2003; Kang et al.
2007), even for a rather large value of $\epsilon_{B}=0.27$, the injection fraction is smaller than $10^{-3}$ and $P_{c, 2} / \rho_{1} u_{s}^{2}<0.01$. So we can safely adopt the test-particle solution for those weak shocks, unless there are abundant pre-existing CRs in the preshock flow.

The middle panels show the cases with the same $Q_{\text {inj }}$, independent of $M$. For these cases, $T_{1}=10^{6} \mathrm{~K}, \delta=1$, and $p_{\max }=10^{6} \mathrm{GeV} / c$. With the same $Q_{\mathrm{inj}}$, the injection fraction is almost independent of $M$ except for weak shocks with $M \lesssim 5$. For $Q_{\mathrm{inj}}=3.8, P_{c, 2} / \rho_{1} u_{s}^{2} \lesssim 0.1$ for all shocks. One can see that $Q_{\text {inj }} \approx 3.8$ is the critical value, above which the injection fraction becomes $\xi \lesssim 10^{-4}$ and the ratio $P_{c, 2} /\left(\rho_{1} u_{s}^{2}\right) \lesssim 0.1$. Hence, if $p_{\text {inj }} \gtrsim 3.8 p_{\text {th }}$, the CR injection fraction is small enough to guarantee the validity of the test-particle solution. But once again one should note that $P_{c} \propto \ln p^{*}$ for strong shocks.

The bottom panels show the cases in which the preshock temperature is $T_{1}=10^{5}-10^{7} \mathrm{~K}$. Since the ratio $P_{c, 2} /\left(\rho_{1} u_{s}^{2}\right) \propto$ $\xi u_{s}^{-1}$ and $\xi$ does not depend on $T_{1}, P_{c, 2} /\left(\rho_{1} u_{s}^{2}\right) \propto \xi T_{1}^{-1 / 2}$ for a given Mach number, $M=u_{s} / c_{s}$. So we chose $\epsilon_{B} \approx 0.20-0.22$ for different $T_{1}$, which results in $\xi \sim 10^{-4}\left(T_{1} / 10^{6} \mathrm{~K}\right)^{1 / 2}$. This gives the similar value of $P_{c, 2} /\left(\rho_{1} u_{s}^{2}\right) \sim 0.1$ for three values of $T_{1}$. For these shocks, the test-particle solution would be valid.

When $P_{c, 2} /\left(\rho_{1} u_{s}^{2}\right)>0.1$, the nonlinear feedback of the diffusive CR pressure becomes important and the evolution of CR-modified shocks should be followed by DSA simulations. 

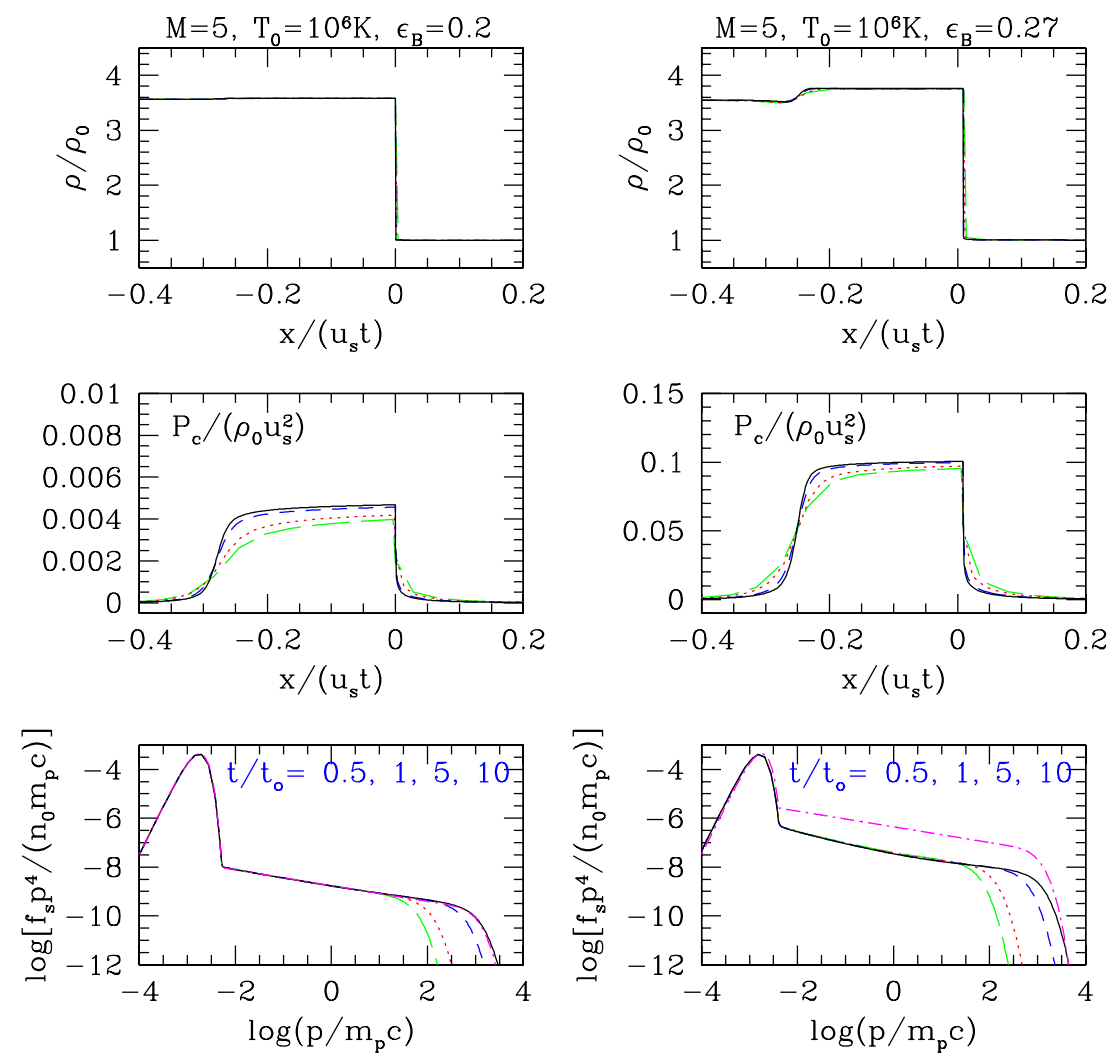

Figure 5. Time-dependent DSA simulation results for an $M=5$ shock for $\epsilon_{B}=0.20$ (low injection rate, left panels) and $\epsilon_{B}=0.27$ (high injection rate, right panels). Here, $T_{1}=10^{6} \mathrm{~K}, v_{A} / c_{s}=0.42$, and $\kappa=10^{-3}\left(p / m_{p} c\right)\left(\rho_{0} / \rho\right)$. At the last time epoch $\left(t / t_{o}=10\right)$, the cutoff momentum becomes $p^{*}=1.2 p_{\max } \approx 10^{3} \mathrm{GeV} / c$. The shock structure is shown at $t / t_{o}=0.5$ (long dashed lines), 1 (dotted), 5 (dashed), and 10 (solid) as a function of the similarity variable, $x /\left(u_{s} t\right)$. The (magenta) dot-dashed lines in the bottom panels represent the test-particle spectra given in Equation (10) at $t / t_{o}=10$. Note that the dot-dashed line (analytic solution) and solid line (numerical solution) almost coincide with each other in the case with $\epsilon_{B}=0.2$. Here, $t_{o}$ is a normalization constant.

(A color version of this figure is available in the online journal.)

Figure 5 compares the evolution of a slightly modified $M=5$ shock $\left(\epsilon_{B}=0.27\right)$ with that of a test-particle shock $\left(\epsilon_{B}=0.2\right)$. In the CR-modified shock, the upstream flow is decelerated in the precursor before it enters the gas subshock. So the quantities at far upstream, immediately upstream, and downstream of the subshock are subscripted with "0," "1," and "2," respectively. For the test-particle shock, $\rho_{1}=\rho_{0}$ and $T_{1}=T_{0}$. Here, $T_{0}=10^{6} \mathrm{~K}$ and $v_{A} / c_{s}=0.42$. The simulations start with a purely gasdynamic shock at rest at $x=0$, initialized according to Rankine-Hugoniot relations with $u_{0}=-1, \rho_{0}=1$, and a gas adiabatic index, $\gamma_{g}=5 / 3$. There are no pre-existing CRs.

The test-particle spectrum given in Equation (10) with $p^{*}=$ $1.2 p_{\max }$ at $t / t_{0}=10$ is also shown for comparison (dot-dashed lines) in the bottom panels. In the test-particle shock with $\epsilon_{B}=0.2$, both $P_{c, 2} /\left(\rho_{0} u_{s}^{2}\right) \approx 0.005$ and $f\left(x_{s}\right)$ from the DSA simulation agree well with the test-particle solution given in Equation (10), as expected.

If we were to take the test-particle spectrum with $\epsilon_{B}=0.27$, we would obtain $\xi=1.74 \times 10^{-3}$ and $P_{c, 2} /\left(\rho_{1} u_{s}^{2}\right)=1.17$, which is unphysical. In the CR-modified solution from the DSA simulation, however, $\xi \approx 3.6 \times 10^{-4}$ and $P_{c, 2} /\left(\rho_{1} u_{s}^{2}\right) \approx 0.1$. The postshock temperature $T_{2}$ is reduced about $17 \%$ in the CRmodified solution (due to higher $\rho_{2}$ and lower $p_{g, 2}$ ), compared with that in the test-particle solution. But $u_{2}$ and so $p_{\text {inj }}$ remain about the same. As a result, the amplitude $f_{\text {inj }}$ is lower than that of the test-particle spectrum (see the bottom right panel of Figure 5) and so the injection rate is reduced in the CRmodified solution. The distribution function $f\left(x_{s}, p\right)$ from the DSA simulation is slightly steeper for $p / m_{p} c<10$ and slightly flatter for $p / m_{p} c>10$ than the test-particle power law, because the flow velocity is slightly modified. This demonstrates that the DSA saturates in the limit of efficient injection through the modification of the shock structure (i.e., a precursor plus a weak gas subshock), which in turn reduces the injection rate. Thus, the ratio $P_{c, 2} /\left(\rho_{1} u_{s}^{2}\right)$ approaches to $\sim 1 / 2$ for strongly modified CR shocks (Kang \& Jones 2007).

Finally, we find that the volume-integrated spectrum contained in the simulation volume can be obtained simply from $F(p)=\int f(x, p) d x \approx f_{\mathrm{tp}}\left(x_{s}, p\right) u_{2} t$. This provides the total CR spectrum accelerated by the age $t$.

\section{SUMMARY}

Although the nonlinear DSA involves rather complex plasma and MHD processes, the test-particle solution may unveil some simple yet essential pictures of the theory. In this study, we suggest an analytic form for the CR spectrum from DSA in the test-particle regime, based on simple models for thermal leakage injection and Alfvénic drift of self-generated resonant waves.

If the particle diffusion is specified (e.g., Bohm diffusion), the shock Mach number is the primary parameter that determines the efficiency of DSA. For a given shock Mach number, the fraction of injected CR particles becomes the next key factor. Since the postshock thermal velocity distribution at the injection momentum determines the amplitude of the powerlaw distribution in the thermal leakage injection model, the ratio $Q_{\text {inj }}=p_{\text {inj }} / p_{\text {th }}$ is the key parameter that controls the CR 
injection fraction and in turn determines the CR acceleration efficiency. On the other hand, as a result of the drift of Alfvén waves in the precursor, the power-law slope should be revised as in Equation (2), which leads to a CR spectrum much steeper than the canonical test-particle power law. This effect is negligible for shocks with the Alfvénic Mach number, $M_{A} \gtrsim 30$.

For shocks with the sonic Mach number $M \gtrsim 10$, depending on the preshock temperature $T_{1}$, the injection fraction, $\xi \lesssim$ $\xi_{c} \approx 10^{-4}\left(T_{1} / 10^{6} \mathrm{~K}\right)^{1 / 2}$, would lead to the downstream $\mathrm{CR}$ pressure, $P_{c, 2} /\left(\rho_{1} u_{s}^{2}\right) \lesssim 0.1$. The exact values depend on other parameters such as $v_{A}$. In that case, the CR spectrum at the shock location can be described by the test-particle power law given in Equation (10), in which the amplitude, $f_{\text {inj }}$, is fixed by the postshock thermal distribution at $p_{\text {inj }}$ given in Equation (4). For SNRs in the hot phase of the ISM with $T_{1}=10^{6} \mathrm{~K}$, for example, the CR injection fraction becomes less than $10^{-4}$, if $Q_{\text {inj }} \gtrsim 3.8$ (or $\epsilon_{B} \lesssim 0.21$ ). For weaker shocks with $M<5$, the test-particle solution is valid even for larger injection fraction, so $\xi_{c}<10^{-3}$.

We have shown that the CR spectrum at the shock location in time-dependent, test-particle shocks without particle escape could be approximated by the analytic solution given in Equation (10), which was derived for steady-state, test-particle shocks by Caprioli et al. (2009), with the cutoff momentum set as $p^{*} \approx 1.2 p_{\max }(t)$. If the CR injection is inefficient, which should be true for weak shocks with $M \lesssim 5$ found in the ICM, the test-particle solution presented in this paper should provide a good approximation. Figure 4 should provide guidance to assess if a shock with specific properties can be treated with the test-particle solution.

With the injection rate greater than $\xi_{c}$, especially for shocks with $M>5$, the spectrum deviates from the test-particle form due to the modified flow structure caused by the diffusive CR pressure. In fact, the DSA efficiency saturates in strongly modified CR shocks, because the postshock temperature gets lower and therefore the injection rate is reduced. Based on the results of the DSA simulations, Kang et al. (2009) suggested that CR-modified shocks evolve self-similarly once the total pressure is dominated by relativistic particles and that the $\mathrm{CR}$ spectrum at the subshock can be approximated by the sum of two power laws with the slopes determined by the subshock and total compression ratios with an exponential cutoff at $p_{\text {max }}(t)$.

The authors thank T. W. Jones for helpful comments on the paper. H.K. was supported by National Research Foundation of Korea through grant 2010-0016425. D.R. was supported by the National Research Foundation of Korea through grant KRF2007-341-C00020.

\section{REFERENCES}

Ave, M., Boyle, P. J., Höppner, C., Marshall, J., \& Müller, D. 2009, ApJ, 697, 106

Bell, A. R. 1978, MNRAS, 182, 147

Blandford, R. D., \& Eichler, D. 1987, Phys. Rep., 154, 1

Berezhko, E. G., Ksenofontov, L. T., \& Völk, H. J. 2009, A\&A, 505, 169

Berezhko, E. G., \& Völk, H. J. 1997, Astropart. Phys., 7, 183

Caprioli, D., Blasi, P., \& Amato, E. 2009, MNRAS, 396, 2065 (CBA09)

Caprioli, D., Blasi, P., \& Amato, E. 2010, arXiv:1007.1925

Drury, L. O’C. 1983, Rep. Prog. Phys., 46, 973

Gieseler, U. D. J., Jones, T. W., \& Kang, H. 2000, A\&A, 364, 911

Jones, T. W. 1993, ApJ, 413, 619

Kang, H. 2010, J. Korean Astron. Soc., 43, 25

Kang, H., \& Jones, T. W. 2007, Astropart. Phys., 28, 232

Kang, H., Jones, T. W., \& Gieseler, U. D. J. 2002, ApJ, 579, 337

Kang, H., Ryu, D., Cen, R., \& Ostriker, J. P. 2007, ApJ, 669, 729

Kang, H., Ryu, D., \& Jones, T. W. 2009, ApJ, 695, 1273

Malkov, M. A. 1998, Phys. Rev. E, 58, 4911

Malkov, M. A., \& Drury, L.O'C. 2001, Rep. Prog. Phys., 64, 429

Malkov, M. A., \& Völk, H. J. 1998, Adv. Space Res., 21, 551

Morlino, G., Amato, E., \& Blasi, P. 2009, MNRAS, 392, 240

Ptuskin, V. S., Zirakashvili, V. N., \& Seo, E. S. 2010, ApJ, 718, 31

Reynolds, S. P. 2008, ARA\&A, 46, 89

Ryu, D., Kang, H., Hallman, E., \& Jones, T. W. 2003, ApJ, 593, 599

Skilling, J. 1975, MNRAS, 172, 557

Zirakashvili, V. N., \& Ptuskin, V. S. 2008, AIP Conf. Proc. 1085, Proc. 4th Int. Meeting on High Energy Gamma-Ray Astronomy, ed. F. A. Aharonian, W. Hofmann, \& F. Rieger (Melville, NY: AIP), 336 